\title{
Vaccine preventable diseases and immunization during humanitarian emergencies: challenges and lessons learned from the Eastern Mediterranean Region
}

\author{
N. Teleb ${ }^{1}$ and R. Hajjeh ${ }^{2}$
}

The last few years have seen the WHO Eastern Mediterranean Region suffer from multiple wars and conflicts leading to humanitarian emergencies of unprecedented magnitude. In addition to the many lives lost and affected, the conflicts have significantly impacted the infrastructure needed for delivery of healthcare services (1). Approximately 30 million people have fled their countries; the refugee population in Jordan has doubled and even tripled in Lebanon (2). The population displacement and resettlement, overcrowding, poverty, poor sanitation, and malnutrition due to food shortages have increased morbidity and mortality from various diseases, notably communicable diseases.

Control of vaccine-preventable diseases (VPDs) is especially vulnerable to disruption of health-care systems due to the need for continuous implementation and monitoring (3). The Syrian Arab Republic had a very strong immunization program prior to its current conflict, with more than 90 percent of children routinely vaccinated and the last polio case reported in 1999. However, diphtheria-tetanus-pertussis (DTP3) coverage in the country dropped to $41 \%$ in 2015; in 2013, less than two years after the start of conflict, the country experienced a polio outbreak that paralyzed 35 children. To contain it, more than 25 million doses of oral polio vaccine were administered. In
2014, other VPDs resurged in the Syrian Arab Republic, including measles and pertussis. Both Jordan and Lebanon faced large outbreaks of measles due to the influx of Syrian refugees. In Lebanon alone, the measles incidence increased from 2.1 to $411 \mathrm{cases} / \mathrm{million}$ population in 2012 and 2013 respectively (3).

TheRegionhasearlierdemonstrated significant progress in measles control, with reported measles cases decreasing from 89,478 in 1998 to 10,072 in 2010 (77\%) (4). However, with the political turmoil and significant decrease in donor funding, regional progress slowed and measles cases doubled to reach 20898 cases in 2015. The regional average of DTP3 coverage gradually declined from $86 \%$ in 2010 to $80 \%$ in 2015 (WHO/UNICEF estimates). In 2015, 3.8 million infants missed their third DTP dose in the Region - more than $90 \%$ of these infants are in conflictaffected countries (EMRO, unpublished data).

\section{Immunization activities during conflict}

Remarkable efforts were devoted to maintaining immunization programs and reaching every child with life-saving vaccines, even under active war and life-threatening situations. During 2011-2016, more than 248 million children aged 6 months to 15 years were reached by measles-containing vaccines in conflict-affected countries as well as countries hosting refugees in the Region (5).

While concerted partners' support was a key factor for accessing required resources, governments' commitment and allocation of national resources was critical. Most importantly, the devotion of health workers and their relentless efforts to reach children in hard to reach areas, and the demand from communities for vaccines, were major success elements. The examples of Yemen and the Syrian Arab Republic best illustrate how concerted efforts at multiple levels are critical to sustain immunization coverage and prevent outbreaks of VPDs.

\section{Yemen}

More than two million people in Yemen were displaced due to conflict and many health facilities destroyed (one third of health facilities and one fourth of vaccine stores). However, pentavalent vaccine (DTP-HepB-Hib) coverage dropped only slightly from $87 \%$ in 2010 to $84 \%$ in 2015 (6). To maintain coverage, outreach activities were conducted in the remote areas $(24,000$ and 30,000 sessions in 2014 and 2015 respectively), in addition to three rounds of polio national immunization days (NIDs) each year, thus sustaining the polio-free status. Yemen also smoothly introduced two new vaccines (measles-rubella $[\mathrm{MR}]$ and inactivated polio vaccine [IPV]), while sustaining other new vaccines (Hib, rotavirus and pneumococcal vaccines). These achievements 
were possible through concerted efforts spearheaded by WHO, effective coordination among partners through weekly meetings attended by all key stakeholders, active utilization of the Health Cluster forum to advocate for immunization, establishing a functional immunization Operational Control Room at central and governorate levels, and the devotion of front-line immunization workers. The effective uses of resources, especially Global Alliance for Vaccines and Immunization (GAVI) support, and distribution of roles and responsibilities among different partners, were instrumental.

\section{Syrian Arab Republic}

When the conflict in the Syrian Arab Republic began in 2012, routine immunization stopped in areas outside recognized government control. During the short break in the conflict in February 2016, WHO and UNICEF in collaboration with the Syrian Ministry of Health and other supporting partners (Syria Arab Red Crescent, Syria Immunization Group, and local NGOs), as part of Accelerated Implementation of Routine Immunization initiative (AIRI), conducted multiple antigens vaccination campaigns in the hard-toreach areas deprived of access to routine vaccines. Every child born since the start of the conflict was provided with three doses of pentavalent vaccine, 3 doses of oral polio vaccine, 2 doses of MR vaccine, and one dose of IPV. Despite the active war in the north of the Syrian Arab Republic, three campaigns were implemented - 1.9 million doses of pentavalent vaccine, more than 1.3 million doses of MR vaccine, more than 1.9 million of OPV and 168,405 doses of IPV (EMRO, unpublished data).

The constant support of the partners, led by WHO and UNICEF, and the high dedication of the local NGOs and health workers were behind the exceptional success in the areas that implemented the campaigns. Independent post-campaign monitoring, using card information or finger marking, proved that more than $90 \%$ of the children were reached - an incredible success in view of all the challenges.

\section{Challenges}

Multiple challenges were faced while supporting immunization activities in the Region during conflict. Many health facilities were destroyed and the functioning facilities faced major challenges with availability of fuel and electricity. Security concerns were major obstacles, affecting all planned activities, particularly outreach and mobile activities, and significantly increasing operational costs. Obviously, the active war in many areas made parents reluctant to take their children to health centres for vaccination. The resulting inadequate number of immunization staff who had fled conflict areas or were reassigned to other healthcare priority functions, was also a major difficulty. Lastly, in many countries, immunization activities were limited by the shortage of funds required for vaccine purchase and operational costs.

\section{Policy level}

The Strategic Advisory Group of Experts (SAGE) and the World Health Assembly requested in 2011 that WHO develops guidance on the delivery of immunization services in humanitarian emergency settings. In 2013, the final version of the framework entitled "Vaccination in acute humanitarian emergencies: a framework for decision making", was released and disseminated (7). The document provides key decision-makers in countries and partner agencies with a systematic and comprehensive approach to decisionmaking on the use of vaccines in acute humanitarian emergencies, taking into account various factors including the epidemiologic risk assessment, vaccine characteristics, and contextual factors. In addition, it provides guidance on ethical concerns such as prioritization of interventions, equity, and informed consent. It is currently being updated to facilitate its use, based on input from various countries affected by conflict. In addition, a package of tools on vaccination in humanitarian emergencies is being prepared, in order to provide practical guidance for delivering vaccines and improving vaccination coverage during these situations, as well as overcoming barriers to timely supply affordable vaccines.

\section{Conclusions}

Sustaining immunization activities and preventing VPD outbreaks during conflicts is very difficult and requires massive and adequately coordinated efforts by all parties involved, including governments and other political parties, since the health of populations and children should transcend all political considerations. Although emergencies often lead to disruption of immunizations and other health services, their impact can be minimized if strong health systems are in place and appropriate preparedness measures are taken preemptively. Partners, whether technical, implementing or financial, play an even more important role in supporting public health programmes overall and immunization in particular during conflicts. Community engagement and demand for vaccines remains essential. Policy frameworks recently developed should serve as guidance for decision-making and implementation. The global community should recognize that the deterioration in the public health situation in the Eastern Mediterranean Region is of an unprecedented and dramatic scale. Communicable diseases, and VPDs in particular, require urgent attention. If adequate support, both financially and programmatically, is not consistently provided, the situation poses an immense threat to health security globally, and to the success of major health initiatives such as polio eradication and measles elimination. 


\section{References}

1. Alwan, A. The cost of war. Newsweek Middle East edition, 5 November 2015 (http://newsweekme.com/the-cost-of-war/, accessed 23 January 2017).

2. Displaced populations. Geneva: World Health Organization; 2016 (http://www.emro.who.int/eha/displaced-populations/index.html, accessed 26 January 2017).

3. Lam E, McCarthy A, Brennan M. Vaccine-preventable diseases in humanitarian emergencies among refugee and internally-displaced populations. Hum Vaccin Immunother. 2015;11:2627-36.

4. Teleb N, Lebo E, Ahmed H, Hossam AR, El Sayed, Dabbagh A et al. Progress towards measles elimination - Eastern Mediterranean Region, 2008-2012. MMWR 2014;63:511-15.
5. Summary of supplementary immunization activities from January 2000 to December 2017. Geneva: World Health Organization; 2017 (http://www.who.int/entity/immunization/monitoring_surveillance/data/Summary_Measles_SIA_ Jan2000_Dec2017.xls?ua=1, accessed 23 January 2017).

6. WHO/UNICEF Joint Reporting form 2015. Geneva: World Health Organization; 2016 (http://www.who.int/immunization/monitoring_surveillance/data/yem.pdf).

7. Vaccination in acute humanitarian emergencies: a framework for decision making. Geneva: World Health Organization; 2013 (http://www.who.int/hac/techguidance/tools/vaccines_in_humanitarian_emergency_2013.pdf?ua =1, accessed 26 January 2017). 